\title{
LA NUEZ Y SU SIMBOLIZACIÓN EN LA EDAD MEDIA LATINA
}

\author{
Francisco PEJENAUTE RUBIO \\ Universidad de Oviedo
}

Los escritores latino-medievales viven inmersos en un mundo de simbolismos que, sobre todo en un afín didáctico, les sirven para explicar a un público, por lo general inculto e ignaro, las verdades de la fe cristiana y el destino del hombre en el mundo. La riqueza de ese mundo en sus manifestaciones simbólicas es inconmensurable. Y en esa simbolización extrema todo sirve: desde las cosas mís palpables y vulgares de la vida cotidiana hasta los objetos y los seres más fantásticos y quiméricos.

Es más, tanto los unos como los otros son tratados con la misma seriedad y con el mismo grado de aceptabilidad; hasta el punto de que no tiene ningún sentido preguntarse si, ni siquiera en su fuero interno, a la hora de la simbolización, distinguían lo real de lo imaginario, lo concreto de lo quimérico. En la simbolización a la que sometían a la naturaleza entera lo importante para ellos era el hecho mismo de la simbolización, o, por mejor decir, el resultado y el fruto de la simbolización. Lo importante no era si el ave fénix existía realmente; ni siquiera lo era si ellos creían o no en su existencia real; lo que les interesaba era que el ave fénix simboliza algo; y csa simbolización de algo era lo que para ellos contaba?

En definitiva, en todos los casos se trata de descubrir, bajo la capa de la materialidad de las cosas, el sentido oculto que ellas encierran y que sólo los doctos adivinan. Toda simbolización es, en suma, un adentrarse. rompiendo la materialidad exterior, en el ntícleo que da sentido transcendente al mundo que nos rodea: es decir, el descubrimiento de la pulpa y de la almendra, una vez que se ha hecho saltar el caparazón que la envuelve.

Por eso, nada más congruente que preguntarse, si de simbolización en la Edad Media hablamos, por el sentido oculto que los autores de esa época descubrieron en un fruto como la nuez que, en su más exacta materialidad, encierra, bajo sus capas y cortezas, un núcleo apetecible y sabroso.

Ahora bien, previamente tenemos que hacer constar que con el término nux los antiguos designaban todo fruto que, con una pulpa en su interior, estaba revestỉdo de diversas capas, más o menos duras y resistentes, lo que motivaba que, con diversas determinaciones adjetivales, se refirieran no

' EI tema de la simbolización en la Edad Madia, sobre el quo existe una nutrida bibliografía, lo hemos tratado, por nuestra parte, en aCreencia, superstición y simbolización en los Bestiarios medievales: el caso del unicorniow, pendionte do publicación en las Actas de las XIV Jornadas de Estudios Clásicos de las Universidades de Castilla y León: Creencias y stupersticiones en el mundo clásico y medieval (León 9-12 de noviembre de 1999). 
sólo a distintos tipos de nueces sino a frutos diversos como la avellana, la almendra, la castaña, la bellota, etc. Una aproximación al mundo de tales frutos bajo el nombre genérico de nax la podemos hacer a través de los textos de autores clásicos y medievales como: Catón ${ }^{2}$, Plinio ${ }^{3}$, Macrobio ${ }^{4}$, san Isidoro ${ }^{5}$, Gilbert de Foliot ${ }^{6}$, etc. Para una distribución sistemática de tales referencias véase el lema nux, nucis en la obra de J. André: Les noms de plantes dans la Rome antique ${ }^{7}$.

Un caso especial es el constituido por el término amygdala ${ }^{\mathrm{s}}$, amygdalus,

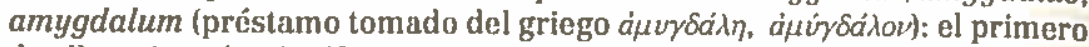
de ellos, sin más, significa, en principio, 'almendra'; a veces, con el mismo significado y en su forma adjetival o como determinación en genitivo, va acompañando a nux (nux amygdalina) o a poma (poma amygdali); es más, hay autores (medievales) que lo identifican con nux (nux sive amygdalum) con el significado (supuesto) de 'nuez', y como quiera que, bien sea en su uso absoluto o acompañado de alguna determinación, ha sufrido el mismo proceso de simbolización que nux (= 'nuez'), cuando se ofrezcan las simbolizaciones de la nuez, se ofrecerán, de igual modo, las de la almendra, dejando constancia de ello cuando se trate de la simbolización de esta última.

Ahora bien, la nuez/almendra ha podido dar lugar a diversas simbolizaciones a partír de diversas circunstancias: por un lado, se trata de un fruto que tiene dos capas y un núcleo; por otro, al núcleo o pulpa sólo se llega tras romper aquéllas ${ }^{y}$; $y$, en tercer lugar, se trata de un fruto amargo y duro por

\footnotetext{
${ }^{2}$ De agri cultura, 8,$2 ; 17,2 ; 48,3 ; 51,1 ; 1331,2 ; 143,3$.

${ }^{3}$ Nat. Hist., XV 86-92.

4 Saturnalia, III 18.
}

5 Etymologiae, XVII 21-23, en texto qua, al pie de la lotra repiten al autor del libro IIl del De bestits et alis rebas, cap. 56 (la obra os una compilación publicada on la Patrologia latina, vol. 177 - al texto en cuestión, cols. 113B-C-entro las obrus de Hugo de san Víctor, aunque Migne. su editor, el libro III se lo atribuye a Enrique do Gante) y Rabano Mauro, De universo, lib. XIX, cap. 514 (111), 514A. Iacemos notar que, aquí, y en lo sucesivo, las referencias a textos do Padres de Ja Iglesia y autores medievales se entiende quo están hechas a partir de la Patrologia Latina, por lo que las citas se harain, directamente, aludiendo a volumen y col(s).

"Expositio in Cantica Canticortum, 202, 1287A.

7 Paris. 1985, pp. 14-15.

${ }^{8}$ El término puedo presentar diversas formus: amigdala. amigdula, amygdola. amigdola. amiddula, amandula, amandola (véaso el Thesaurus Linguae Latinae o.J. Andró, o. c., p.14l.

"La idea de que, si se quiere llegar a la pulpa del fruto, hay que romper la nuez se convirtió on un proverbio que, recogido, por primara vez según creemos, por Plauto (Carculio, 55: "qui e nuce nuculeum esse toll, (rangit nucem") tuvo un amplio eco. Los compiladores de proverbios lo han registrado, unas veces al pie de la lotra, otras, con variantes: asi. $\Lambda$. Otto. Die Sprichwörler der Hömer, Hildesheim, 1965 [= Leipzig, 1890). s. v. nux, quien, a continuación del texto de Plauto ofrece uno de san Jerónimo, Epistulac, 58, 9 (22, 585): "Qui edero vult nucleum, frangat nucem", que 
fuera y dulce por dentro, siendo mucho más numerosas las simbolizaciones en los dos primeros casos que en el tercero".

La nuez va a gozar de una simbolización generalizada entre los autores medievales; ahora bien, en el mundo greco-romano este fruto ya se habia cargado de una cierta dosís de simbolismo, al que se había llegado a través de lo que podríamos denominar una sinécdoque simbolista: por lo que se refiere al mundo romano (al que los autores medievales tenían un acceso directo). del hecho de que las nueces estaban en la base de numerosos juegos infantiles o se repartían (junto con otros frutos y golosinas) entre amigos y lamiliares en determinadas fiestas, se pasó a un cierto grado de simbolización de las mismas que se manifiesta en cómo se aludia a ellas entre las canciones subidas de tono (versos fescenninos) en los cortejos nupciales. Veamos algunos textos confirmatorios y aludamos a algunas circunstancias pertinentes:

luego repetirán los nutores medievales; por gemplo, Lupo de Olmeto, Regula monachorum ex scriptis Hieronymi collecta, 30, 365C: "Qui edore vult nucleum, Prangat nucem"; "Auctor incertus (Hieronymus Stridonensis?). Commentarius in Marcum, cap. I (30, 595C-D): "quj desiderat nucloum, frangit nucem"; "Auctor incertus" (Augustinus llipponensis?), Ad fratres in eremo commorantes, Sermo XLl; "De observantia jejunii quadragesimalis, 40, 1314: "frangatis nucem, ut accipiatis nucloum"; Walafrido Estrabón. Evangelium secundum Marcum, 114, 182B: “Qui desiderat nucleum, nucem frangat": Paulo Alvaro, Epistolae. Epistola IV "Joanni Spalensi", 13, 121, 435c: "Qui esse vult nucleum, frangit nucem": Ruperto Tuitiense, Commentarius In Librum Ecclesiastes, 168, 1210B: "Qui edere vult nucloum, frangat nucern"; Guerrico Igniacense, Sermones per annum. "De resurrectione Domini", Sermo I, 2. (185, 142A): "Irangite testam et invenietis escam"; Ricardo Cenomano, Prologus in Magistrum IPetrum Lombardum/ sive collatio diversarum translationum. 191, 38A: "Qui edere vult nucleum. Irangat nucem"; o Zacarías Crisopolitano, De concordia evangelistarum, 186, 111B: "Qui desiderat nucleum, nucem frangat". Igualmente, H. Walther, en Carmina Medii Aevi. Band 2; Proverbia Sententiaeque Medii Aevi, Gottingen, 1963. $\mathrm{N}^{\mathrm{n}} 23818$ y 36497, recogiendo al pio de la letra el dicho de Plauto, y, con variantes, en el 9914: "Frange nucis tegmen, si cupis esse nucem"; el 9914: "Inspice, quid lated!! Nucleus latot in nuce. granum / Si placet, a stipulis eliciatur, adest"; el 24063: "Qui e nuce nucleum esse vult, Irangat nucem", ó 39847g6: "Qui nucem esse vult, oportet frangat ante nucleum". La idea de que hay que partir (golpoar) la nuez para que ofrezca su fruto, está recogida en el No 27016: "Rusticus ac asinus, nux, hec tria connumerata, /Non faciunt fructum, fuerint nisi combaculata"; $y$, en variante misigina, en 34954 : "Asinus, mulier, nux nil recte faclunt, si verbera cessent".

${ }^{14}$ Gran parte do las simbolizaciones de la nuez/almendra surgen a propósito de comentarios a ciertos pasajes de la Vulgata. Se trata de los siguientes: Cantica Canticorum, 6,10: "Descendi in hortum nucum, ut viderem poma convallium"; Numeri. 17,8 (la seca vara de Aurón florece y da frutos -amygdalae-): "sequenti dio regressus invenit germinasse virgum Aaron in domo Levi: et turgentibus gemmis eruperant flores, qui, foliis dilatatis, in amygdalas deformati sunt"; Exodus, 25, 33; $25,34: 37,19: 37,20$ (cómo deben ser las copas del templo): "Tres scyphi quasi in nucis modum", y Ecclesiastes, 12, 5: "florebil amygdalus". 
1. nuces relinquere = 'abandonar los juegos infantiles" /"salir de la infancia': testimonios al respecto los podemos encontrar en diversos autores clísicos; asi, Catulo" ${ }^{11}$ Persio ${ }^{12}$, Fedro ${ }^{13}$, o Marcial $^{14}$.También entre los autores medievales abundan referencias a las nueces como parte integrante de los que, en un tiempo, fueron juegos infantiles: por ejemplo, Felipe de Harveng ${ }^{15}$; y este mismo autor vuelve sobre la misma idea en otro pasaje ${ }^{15}$.

2. Las nueces, entre los niños romanos, venían a ser como las canicas entre los nuestros; de muchos y diversos juegos entraban a formar parte, y en no pocas ocasiones, por dejarse llevar de su desmesurada afición a tales juegos, al parecer inocentes, más de una vez algún niño salía con las nalgas calientes ${ }^{17}$. Horacio, por su parte, nos presentu a un joven apasionado por los juegos de dados y nueces, dados y nueces que llevaba en los pliegues de su túnica". Hasta el propio emperador Augusto, tan aficionado, como se sabe, al juego de dados, jugaba a las nueces con los rapazuelos ${ }^{19}$.

3. El poema $N u x$, falsamente atribuido a Ovidio, describe ${ }^{20}$ no menos de siete juegos con nueces. Ahora bien no sólo los niños jugaban con ellas; también los mayores se divertían con nueces, en especial tomándolas como elementos de apuesta en diversos juegos ${ }^{21}$.

4. Entre los obsequios que se intercambiaban en las fiestas de las Saturnales no solian faltar las nueces, calificadas, en ose caso, como nuces Saturnalitiae ${ }^{22}$. nucibus (...)"

${ }^{1}$ Catulo, 61, 131, 3-5: "Da nucem pueris, iners/concubine: satis diu/lusisti

12 I 10: "ac nucibus facimus quaecumque relictis".

${ }^{13}$ III 14 (=n" 58), 1-3: "Puerorum in turba quidam Judentem Atticus / Aesopum nucibus cum uidisset, restitit, / et quasi delirium risit".

" V 84, 1-2; "Iam tristis nucibus puer relictis/clamoso rouocatur a magistro".

15 Epistolac. "Epistola III ad I. Ieroaldum", 203. 26B: "Neque ut arbitror indigne feres si coluphum tibi fidenter incutiam, quo incusso, velut tenenti nuces exculiam: ut axcussis quat puerilia surt ot plona vanitatis, solummmodo tenoatur codes maturior honestae veritatis".

16. "Non projicit nuces et talos, id est vana ot ludicra, quae pueris aetas sua videtur indulsisse"

${ }^{17}$ Marcial, XIV 19(18).1-2: "Alea parua nuces etnon damnosa uidetur:/suope tamen pueris abstulit illa natis".

${ }^{18}$ Sat. , I1 3, 171-2: "Postquam te talos, Aulc, nucesque/ Corre sinu laxo, donare ot ludare uidi".

195 Suctonio, Diuns Augustus, 83, 2: "Animi laxandi causa modo piscabatur hamo, modo talis aut ocellatis nucibusque ludebat cum pueris minutis".

20 Vors. 73 ss.

21 Veamos algunos textos de Marcial: en V1 66, 16: "aleae sed parcae sola luere nuces"; XIII 1, 7; "haec mihj charta nuces, haec est mihi fritillus"; "XIV 1, 12; "Lude', inquis, "nucibus" ; XIV 185, 2: "ne nucibus positis ARMA VIRUMQUE legas".

${ }^{22}$ Expresión que encontramos, por ejemplo, en Marcial, V 30, 8 y en VII 91, 2. 
5. En las bodas, en las que, mientras el novio conducía a la novia al domicilio conyugal, se arrojaban nueces a los niños, que se agolpaban a su paso, no dejaba de aludirse a las nueces en las canciones subidas de tonos con que se acompañaba a los esposos ${ }^{23}$, como se ha apuntado más arriba.

6. Las nueces siguieron constituyendo una de las golosinas que más atraían a los nin̄os de épocas posteriores: san Agustín, en diversos pasajes, nos lo recuerda ${ }^{24}, y$, repitiendo las palabras del santo, Zacarias Crisopolitano ${ }^{25}$; san Bernardo añade el detalle de que a los niños son sus madres las

${ }^{23}$ Catulo, 61, 128-130: "Nec nuces pueris neget/desertum domini audiens / concubinus amorem*. Lechantin di Gubornatis, al comentar el pasaje, remite a Servio, ad Verg, Ecl. 8, 29-30 (el texto de Virgilio es: EcL. VIII, 29-30. "Mopse, nouas incide faces: tibj ducitur uxor; / sparge, marite, nuces"). ("Sparga, marite, nuces", forma parte del verso $73 \mathrm{del}$ Cento muptialis de Ausonio). Dice Servio (según su editor, VIII 29, pero el verso de Virgilio es el 30): "Modo tamen ideo ait 'spargo marite nuces", ut eum culparet infamiac: nam meritorii pueri, id est catamiti, quibus licenter itebantur antiqui, recedentes a turpi servitio nuces spargebant, id est ludum pueritiae, ut signilicarent so puerilia cuncta iam spernere". Y, un poco más adelante, dice el mismo comentarista: "dicitur etiam ideo a novo marito nuces spargi debere, quod projectae in terram tripudium solistimum faciant, quod auspicium ad rem ordiendam optimum est: vol ideo a pueris aspergendas nuces cum strepitu et convicio Magitari, ne quid nova nupta audiat adversum, quo dies nuptiarum dirimatur" ( $A$ propiciar un buen augurio hace referencia Philargyrius, en su comentario al pasaje de Virgilio: "Sparge marite nuces' idest nocte nuptiarum solent nuces iacere, ne perveniat malum augurium"). L.echantin también ofrece el testimonio de Paulo Festo, 179, 8: "nuces llagitantur nuptis ot jaciuntur pueris ut novae nuptae intranti domum novi mariti secundum liat auspicium". El mismo Catulo, on uno de sus epitalamios, menciona repetidas veces a las nueces: unas, aludiendo a la ceremonia nupeial; otras, con referencia, indirecta, a los juegos de los niños con nueces: 61. 126 ss.: "Ne diu tareat procax / Fescennina iocatio, / Nec nuces pueris neget / Desertum domini audiens / Concubinus amore. / Da nuces pueris, iners / Concubina; satis dju/lusisti nucibus; lubet/ lam servire Talasio. / Concubine, nuces da ..."; y v. 140: "Concubine, nuces da". Por su parte, Plinio, Hist. Nal., XV 86, hace reforencił a cómo, durante el cortejo nupcial, se entonaban cancionus licenciosas (versos fescenninos) y se arrojaban nueces a los niños: "ipsao I= nuces| nuotialium Fescenninorum comites". $A$ las nueces del día de bodus hay alguna referencia en alguno de los Proverbia sententiaeque ... recogidos por H. Walther (véase nota 9); por ejomplo el n' 183853 : $^{\circ}$ Linque, marite, nuces, quoniam nunc competit uxor: / Est ultra vires, quam vis agitaveris, hic flos!".

24 In Joannis Evangelium, Tractatus CXXIV, 35, 1609: "Nuces puero demonstrantur, et trahilur"; Enarrationes in Psalmos, 36, 931: "Numquid quontam puero dantur quaedam puerilin, ludicra quibus puerilis animus avocetur, propterea grandeseenti non ci cxcutiuntur e manibus, ut aliquid jam utilius tractet, quod grandem deceat? Verumtamen tu ipso dedisti fitio tuo, el nuces parvulo, et codicem grandi"; Sermones de Sanctis. "Sermo CCCIl", cap. I $(38,1385)$ : "Quaedam enim plerumque parva et tudicra concedit pater parvulis fillis, quae maxime, nisi acceperint, plorant. Banigna et paterna indulgentia haec impertit, haec donat, quas non vult permanero in filiis suis jam grandiusculis, jam proficientibus. Donal orgo puoris nuces (...)".

${ }^{25} \mathrm{De}^{\mathrm{t}}$ concordia evangelistarum, 186, 252B: "llamum viridem ostendis ovi, et trahis jllam. Nuces monstrantur puero, ot trahitur". 
que les cascan la nuez y les dan la almendra ${ }^{26}$.

7. Como resumen digamos que las nueces venian a constituir uno de los elementos que no podían faltar entre los obsequios más comunes ${ }^{27}$.

\section{Simbolización (cristiana) de la nuez/almendra}

\section{1.- La simbolización de la nuez presentada de modo singular}

La nuez, a partir de san Ambrosio y un auctor incertus que a veces se ha identificado con san Agustín (como veremos) va a ir enriqueciéndose, en su simbolización, de una manera progresiva, adquiriendo nuevos matices y nuevas variantes, hasta convertirse en una simbolización trivial, llegăndose al punto de que tal simbolización es ofrecida, en determinados contextos, de una manera casi automática. Ahora bien, hay casos en los que los autores personalizan e individualizan tal simbolización, presentándola en unas circunstancias que hacen de su aparición algo verdaderamente personal. Y precisamente por tales apariciones vamos a comenzar la exposición de los textos:

a) Rabano Mauro hace referencia a la simbolización de la nucz a propósito de la parábola del sembrador: el Señor enseña por parábolas, como una nutrix que presenta a sus pupilos una vasija llena de nueces; ante el asombro de ellos, coge una y, rompiendo la císcara, les muestra el dulce fruto interior; ahora bien, para llegar al fruto hay que romper, previamenter su caparazón. Así hace el Señor con sus parábolas ${ }^{2 \mathrm{H}}$.

b) Un auctor incertus, identificado (con interrogación) en la Patrologia Latina como "Guillelmus de Campellis", en su Dialogus inter Christianum et Judaeum de fide catholica ${ }^{2 y}$, y en su papel de cristiano, trata de

${ }^{26}$ In coena Domini. Sermo "De baptismo, sacramento altaris et ablutione pedum", 183, 271B: "Neque enim tradit mnter parvulo nucem integram, sed frangit cam, ot nucloum porrigit".

${ }^{27}$ Ovidio, Ars amatoria, II, 267-8, dirá: "Adferat alt uuas aut quas Amaryllis amabat / (at nunc castancas non amat illa) nuces".

${ }^{28}$ Commentarium in Matthaeum, I. IV, c. 13 (Pat. Lat. . 107, 944 B-C): "Observa quod (...) parabolam suam Dominus per semetipsum exponit (...). Ad similitudinem utique facitnutricis, quae vas plenum nuejbus, portans alumnis suis alTert; illis autem mirantibus, et propter novitatem quid utilitatis habeat ignorantibus, tollit ipsa primum nucem, of frangens enucloal, ostenditque quam dulcem fructum intus habeat, et quam suavem cibum gustantibus reddat, si quis corticem illius foris rumpat. Ita et Dominus noster adhuc rudibus discipulis parabolam suam primum exponit. et quid sub velamine litterarum dulcedinis habeat ostendit".

${ }^{29}$ Los simulados Diálogos entre un cristiano y un judío acerca de distintos aspectos de la religión cristiana frente a la judaica, proliferan en los siglos XI y XII, pero ya encontramos un precedente en el s. V: Evagrius Gallus, Altercalio Legis inter Simonem Judaeum et Theophilum Christianum (Pat. Lat., 29, 1165 ss.). Recordemos los de Petrus Damianus, Dialogus inter Judacum inquirentem et Christianum respondentem (ibid., 145, 57 s5.), Petrus Alplıonsus, "Dialogus Petri (...) et Moysi Judaci" (Jbid., 157, 537 ss.), Guillelmus de Campellis (?) (que provoca esta Nota) (ibid., 163,1045 ss.). Rupertus Tuitiensis, Annulus sive dialogus inter Christianum et Judaeum (ibid, 170, 559 ss.) y Petrus Abaelardus, Dialogus inter Philosophum. Judacum et Christianum (ibid., 178, 1609 ss.). 
hacer ver a su contrincante que lo que estaba oculto en la Vieja Ley ha quedado desvelado en la Nueva, por lo que ya no se puede vivir bajo aquélla, sino según los dictámenes de ésta. Es más, los dos Testamentos no concuerdan, como pretende el Judío, por lo que no vale servir al uno o al otro, y para que la cosa quede clare, el cristiano le pone un ejemplo. El diảlogo sigue este derrotero: "Cristiano: Supón que tienes una nuez en tu mano.-Judio: Sea, la tengo.- C.: Si pretendieras comerla sin romperla, tal vez te ahogarías.- J.: Indudablemente, eso ocurriría al instante.- C.: Luego la nuez, entera, no es buena para comerla.- J.: Indudablemente.C.: Asi, pues, conviene romper la corteza previamente y así llegar al núcleo.- J.: No hay duda de que no se puede hacer de otra manera.- C.: Escucha, pues: no se puede de buenas maneras comer la nuez entera, ni llegar a su pulpa si antes no se rompe su caparazón, como no puedes llegar a la Ley Nueva si la Vieja no es quebrantada, pues recuerdas que te he dicho muchas veces que la Ley Vieja tenía encerrada, dentro de sí, bajo el manto de figuras y enigmas, la Ley Nueva $(. .)^{\text {no }}$.

Pedro "Comestor", amparándose en la autoridad de J. Escoto, sostiene que la Escritura habla al hombre deividus, no al pedisequas; este último es el que no puede conocer a Dios a través de la comprensión de las Escrituras, cosa que sí hace el deividus, sino que descubre sus huellas a través de las cosas visibles de este mundo; y, para dejar claro su pensamiento, nos invita al banquete en casa de un hombre rico, banquete en el que, tras degustar suculentos platos de alto precio y estando ya los comensales casi saciados, el paterfamilias, para romper la monotonía, les ofrece un plato humilde y de bajo precio: unas nueces. Ahora bien, la nuez tiene un caparazón (testa) no comestible, pero en su interior posee una almendra comestible y dulce, suave a la hora de comerla. Rompamos, pues, la nuez, para llegar a la almendra; arrojemos las hojas para encontrar el fruto ${ }^{31}$.

31" "Ch.: Propono te tenere nucem in manu tua.- J.: Fiat, teneo nucem.- Cr.: Si hanc nucem infractam ederes, forsitan te strangulares.- J.: Utiqua cito contingeret.Cl1.: Ergo nux integra non est bona ad comedendum.- J.: Utique.- Ch.: Prius ergo oportet testam frangere et sic pervenire ad nucleum.- J.: Nullatenus aliter esse potest.Ch.: Audi igitur: non potes nucem integram edere utiliter, nec pervenire ad nucloum nisi prius lesta frangatur, sicut non potes perventre ad novam legem nisi votus tex conquassetur: nam snope dixisse me recolis, quod velus lex novam intra se tenebat inclusam sub figuris th aenigmatibus positam (...) , (Pat. Lat., 163, 1048C-10549A).

"Sermones. Sermo "In capite jojunii in die Cinerum", 198, 1749B-C: Dicit Joannes Scotus: Sic solet Scriptura loqui deivido generi humano, non pedisequo. Est namque, sicut ipse dicit, duplox gonus hominum, podisequum, et quoddam deividum. Podisequum genus hominum est, qui Deum cognoscero non possunt per intelligentiam Seripturarum, sed vestigia cjus quoquo modo indagant per visibilia hujus mundi (...) sed [deividus] Deum videt per intelligentiam Seripturarum (...) Nobis igitur ad mensam divitis opulentam sedentibus, quasi jam saturatis, diversis et pretiosis forculis, ad sublevandum fastidium proponit paterfamilias parvum et vile ferculum, supplo nucem. Sed nux testam habet inedibilem, ot nucleum intus edibilem ot dulcem, 


\section{2.- Hacia una trivialización de la simbolización cristiana de la nuez}

El primer autor que hace de la nuez una simbolización cristiana es, a nuestro entender, san $A$ mbrosio y, tras èl, serán numerosos los Padres de la Iglesia y autores medievales que seguirán su camino: unos verán en la nuez los mismos rasgos a la hora de aplicar la simbolizacion; otros, por su parte, aplicarán las propiedades de tal lruto a un simbolismo distinto; bueno serä. por consiguiente, distinguir tales aplicaciones según sean los rasgos que caracterizan, en cada caso, las simbolizaciones, comenzando por la visión de san Ambrosio ${ }^{32}$ :

2.1. Nuez = lección profética y gracia sacerdotal: $\operatorname{san}$ Ambrosio ${ }^{33}$.

2.2. Nuez $=$ "ius quoddam et summa prophetiae": $\operatorname{san}$ Ambrosio ${ }^{34}$.

2.3. Nuez = en la Iglesia de Cristo, lin doctrina de la Ley y los Profetas: "Auctor incertus" [= s. Agustín? $]^{3,5}$; Rabano Mauro repite, al pie de la letra, el mismo texto ${ }^{36}$.

2.4. Nuez = la ley bajo la que late el espiritu vivilicador: Ruperto Tuitiense $^{37}$.

ad comedendum suavem. Frangamus ergo nucem, ut accipiamus nucleum; projiciamus folia, ut inveniamus fructum".

${ }^{32}$ Hagamos notar que, a veces, un mismo autor propone varias (y distintas) simbolizaciones de la nuez. por lo que a dichos autores los podremos encontrar en diversos apartados on nuestra exposición.

${ }^{33}$ Commentarium in Cantica Canticorum, 14-15 (15, 1944C-D): "Invilatur denique Dej Verbum in lortum nucis, in quo fructus prophetiae lectionis, et sacerdotalis est gratia, quan amara in tentationibus, dura in leboribus, in vilutibus interioribus fructuosa est*. Este mistno texto lo volvemos a encontrar en el tratado De virginitate, $98(16,291 \mathrm{~B})$.

${ }^{34}$ Apologia altera Prophetac David, cap. X (14, 907D-908A): a propúsito del texto "sume tibi baculum nucinum" que, seguin el santo, pertenece a Jeremias. I 11 (tal texto no estai registrado en la Vulgata), se nos dice que: "ot alibi virgam nucinam logimus, per quam intolligimus jus quoddam ot summam prophetias, quae more nucis foris amara in cortice: dura in medio testa, tenera intus est fructuosa. Essi igitur in historia amariora auditis, in typo dura cognoscitis; sperate tamen in mysterio fructuosa".

${ }^{35}$ En un toxto on que las amigdalae son identificadas con las nuces: Sermones slappositii de Scripturis. Sormo "De virga Aaron", 39, 1805-1806): "Quis autem iste est fructus quem attulit? Amygdalac, inquit: amygdalae fratres, nuces sunt. Qui fructus $[=$ лux] primo quidom indumento amarus est, sequonti munitur ac tegitur, tertio sumentem pascit ac nutril. Talis orgo est in Ecclesia Christi doctrina Legis ot prophetarum. Prima littorae lacies satis amara est, quae circumcisionem carnis praecipit, quae de sacrificiis mandat (...). Ilaec omnia, tanquam amarum nucis corticem, projice. Secundo in loco ad munimenta tecta pervenjes, in quo vel moralis doctrina vel ratio continentiae designatur (...). Tertio autem loco, reconditum Ivelut in rucel invenies secretum mysteriorum sapientias et sciontine Dei sensum, quo nutriantur el pascantur animae sanctae (...) $)^{\mathrm{H}}$.

Th Enarrationes in Librum Numerorum, 108, 688C-689A.

${ }^{37}$ Commentaria in Cantica Canticorum, 168, 937C-D: "Donique sicut nux sub amaro cortice, intra testam duram, dulcom et ad vescendum suavem continet 
2.5. Nuez = "correptio et labor continentiae": Beda ${ }^{37}$; se trata de un texto que Martín de León glosa casi literalmente ${ }^{3 y}$ y Rabano Mauro repite al pic de la letra ${ }^{40}$.

2.6. Nuez $=$ pecado/virtud: Hildeberto de Lavardin ${ }^{41}$; "Auctor incertus"

2.7. Nuez = la vida presente: "Auctor incertus" (= Casiodoro Vivarien-

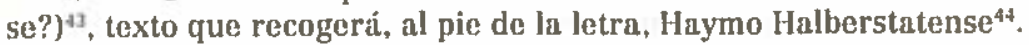

2.8. Nuez = el género humano (el hombre): Volberón de san Pantaleón ${ }^{45}$.

2.9. Nuez $=$ el mundo: "Auctor incertus" $"$ ".

nucleum, ita sub amara voce vel occidente littera, ot durissimo jugo caeremoniarum, suavem alque vivificantom continet spiritum (II Cor. III)".

${ }^{38}$ En un texto en el que la simbolización sirve para nux y para amygdalum: Allegorica expositio in Cantica Canticorum, 91, 1185B: "Potest et ita intellegi: Quin sicut nux, sive amygdalum, amarissimum habet corticem, et testa durissima circumcingitur, et detractis austerioribus et duris, fructus dulcissimus reperitur, sic omnis correptio et labor continentiae, qua sancta exercetur Ecclesia, amara quidem videtur ad praesens, sed fructum parit in futuro dulcissimum".

${ }^{34}$ Sermones. Sermo decimus sextus, "De passionte Domini", 208, 794B-C: "et quomodo nux sive amygdalum amarissimum habet corticem, testam durissimam, ut detractis duris el amaris, frucus dulcissimus reperiatur; sic omnis correctio et labor continentiac amara sunt ad praesens, sed fructum pariunt in novissimis dulcissimum".

* Commentaria in Jeremiam, 111, 80613.

4 Sermones, Sermo LXXV, "In festo omnium Sanctorum", sormo tertius, 171, 706D: "Nux quatuor habet; tres cortices, ct nucleum, qui cum illis nut non comeditur, aut minus dulciter sumitur. Est cortex exterior nativus terrustris, amarus, cum quo nucleus non comeditur, a corrigente peccatum, cum quo nemo salvatur, aut Christo incorporatur. Secundus cortex, ligneus et durus, cum quo idem nucleus non comeditur, id est criminale peceatum, quod nisi tollatur, nemo ad salutent admittitur. Tertius cortex interior tenuis. id est veniale peccatum, quod nisi auferatur, nucleus minus sapide manducatur. Ut ergo in sua dulcodine nucleus sumatur, diligentor ab his tribus amundetur".

42 Expositio in Cantica Canticortm, 172, 583C: "In nuce tria notantur, amarum exterius in cortice, inlra quam testa (......... laguna en el original) carnis ex animu Conditoris dulcor est virtutum".

43 Expositio in Cantica Canticorum, 70, 1092B: "Hortus nucum est vita praesons. Nam sicut in nuce tegmen quidem durissimum vidatur, sed nucleus latet: ita in praesonti vita nostrae conscjentiae clauseae sunt, ot non apparent, donec fracta Lesta corporis manifestentur".

" Commentarium in Cantica Canticorum, 117, 340C

45 Commentaria in Cantica Canticorum, 195, 1220A-B: "I lortum nucum universitatom generis lumani accipere non videtur absurdum, in nuce enim tria considerantur, cortex, testa et nucleus; in humano autem genere constat homo carne, osso et anima; carnis namque materies est quasi cortex, virtus voro ossoa est quasi testa, porro anima du]cedo est quasi nucleus: et sicut nucleus cortice et lesta tegitur. jta anima carne et ossibus vestitur".

th De promissionibus et praedictionibus Dei, $51,779 \mathrm{C}$ : "In nuco vero mundum Ecclesiamque in mundo ostendi, qui pie quaerit, intelligit. Ex duabus quippe partibus conjunctis, tamquam intra cameram coeli et spatia terrae, velut quatuor partos orbis, intrinsecus cum populis continentur: tonue quoddam corium habons in medio sui, ut maria diffusa per partes". 
2.10. Nuez = la Esposa del Cantar de los Cantares: Felipe de Harveng ${ }^{4}$.

2.11. Nuez = Sagrada Escritura: Justo de Urgell ${ }^{45}$; Hicmaro de Reims ${ }^{43}$; Angelomo de Luxeil ${ }^{50}$; Honorio de Autun ${ }^{51}$; Ricardo Cenomano ${ }^{52}$.

2.12. Nuez $=$ el pueblo judio: Volberón de san Pantaleón ${ }^{53}$.

2.13. Nuez = la Virgen Maria: Alain de Lille $e^{54}$.

2.14. Nuez = la Iglesia y sus diversos componentes (fieles de todo tipo:

\$7 Commentaria in Cantica Canticorum, 203, 456C-4574: "Hortus nucum Virgo est, in quem se descendere Sponsus dicit (...). In nuce cortex exterior non mediocriter amarescit, sub cortice testa dura, sed tamen fragilis delitescit; clausus vero sub testa nucleus comedenti suavius indulcescit. Hortus itaque nucum est, cum passionum et tentationum amaritudine tribulatur (...). Hortus est, cum velut testa durjor mira fortitudine praemunitur (...). Nucleus autem absconditus, interna est. suavitas animae diligentis, quae seductos malitia latet oculos porsequentis, quae fracta testa non aufertur, sed defertur quasi manibus offerentis, ot praesentatur Sponso, et ejusdem mensae vel convivio praesidantis".

${ }^{48}$ Explicatio in Cantica Canticorum, 67, 985C: "Hortus nucum, canonem Veteris et Novi Testamenti designat: species enim nucum aliud ostendit in cortico, et aliud retinet in medulla. Quod si quis in Scripturis sanctis non distroverit, et quodammodo velut in nucis corticibus interna non quassierit, ot ad partum speritualis intelligentiae pervenire non potest".

4" Testimonia veterum de s. Ficronymo, 22, 220: "Et sanctus Hlicronymus (...), qui ut dicitur nucem juxta nucleum frangens, medullas et ipsa viscera Seripturae sanctae investigando, Domino inspirante penetrare promoruit (...)".

su Simbolización de poma amygdali: Commentarius in Genesin, 115, 212B-C: "Et sicut quidem poma amygdali tria continet in so, hoc, est corium, testam ot nucleum; ita sacra Scriptura, quam Judaca plebs percepit, trinam continet in se Intelligentiam, id est physicam, ethicam, et logicam, loc est historialem, allegoricam. et moralem significationem".

"1 Expositio in Cantica Canticorum, 172,466B: "Nux etiam est sacra Scriptura, cujus cortex vol testa est littera; nucleus vero spiritualis intelligentia".

${ }^{52}$ Prologus in Magistrum [Petrum Lombardum] sive collatio diversarum translationum.. 191, 38^: “Totum quod legimus in divinis libris, nitet quidom, et fulget atiam in cortice, sod dulcius in medulla cst. Qui edero vult nucleum, frangat nucem"

${ }^{53}$ Commentaria in Cantica Canticorum, 195, 1219B: "hortus enim nucum non inconvenienter dici potest gons Judaeorum: sicut enim in nuce sub cortice at testa nucleus tegitur, ita apud Judacos spiritualis intelligentiae dulcedo sub duritia litteralis suporficiei absconditur. Illa ergo gens hortus ducum dicitur, quja quamvis propter legalium utilitatem praeceptorum et observationum (...) hortus dicatur, omnia tamen futura Ecclesiae sacramenta, in eo sub ligura, quasi sub nucis cortice latebant".

${ }^{54}$ Elucidatio in Cantica Canticorum, 210, 95B: "(...) quod eleganter convonit Virgini gloriosto. Nux quippe labet amarum corticem, testam duram, nucleum dulcissimum; ita Virgo mater exterius habuit infirmitatem el duram testam, id est fidei firmam constantiam, et virginitatis perseverantiam, et du]cissimum nucleum Cliristum quita mundo parit Christum, ex quo data est nobis dulcedo atotornao beatitudinis". 
pecadores, justos, santos, mártires): Gregorio Magno ${ }^{55}$; Beda ${ }^{56}$; Rabano Mauro, por un lado, recogiendo, también aquí al pie de la letra, un texto anterior: en este caso el de Beda ${ }^{57}$; por otro, en un pasaje propio ${ }^{5 \mathrm{H}}$; Walarrido Estrabón $^{5 t 1}$; Anselmo de Laon ${ }^{(t)}$; Ruperto Tuitiense ${ }^{61}$; Bruno Astense ${ }^{(12,}$;

5s Expositio super Cantica Canticortum, 79, 531C: "Quid per nuces, nisi perfoctos quosque intelligimus, qui dum divinam sapientiam intra corpora sua retinent, quasi nucleum in fragili testa portant? Sunt enim quamplures in Ecclesia. quj assidue divinae Soripturae intendunt, quam suavis sit Dominus gustantes, amplius gustare concupiscunt, sancta gaudia in corde ruminant, et ruminantes magis ac magis conval escunt; et tamen for is nescientibus cos, viles apparent; quia ignoratur quam suavem in suis interioribus cibum portent. Quid isti, nisi nuces existunt, qui nuclei dulcedinem intus ferunt, exterius vero carnis vilitatem pratendunt?"

* De Tabernaculo et vestibus sacerdotum, 91, 416D: "Nucem in figura praesentis Ecclesiae poni solere, testatur Salomon (...). Sicut onim dulcem quidem habet fructum interius, sed non hunc ostendere foris, nisi fracta testae duritia, potest: sic justorum vita praesens, ita suavitatem gratiae spiritualis intimo corde conserval, ut haec a proximis quanta sit, nullatenus valeat perspici, donec, soluto corporis domicilio, libere se animae ipsorum alterutrum in coelesti Juce conspiciant, et quanta singulae gratia sancti Spiritus fulgeant, quantum unaquaeque diligatur ab altera, nulli prorsus remaneat occultum".

${ }^{5}$ Commentariat in Exodum 108, 152A-B.

${ }^{58}$ De universo, XIX, $6(111,514 A-B)$ : "Mystice autem nucis arbor significat ecclesiam vel sanctum virum dulcedinom fructuum virtutum in abdito cordis gestantem. (...) Hortus etenim nucum occlesia est praesens, ubi nostras conscientias altarutrum minimo videasnus: sed fracta per tentationem testa corporis, apparebit internae dulcedinis gustus".

5y Liber Exodus, vers. 33 (113, 26913): "Nux Ecclesiam significat, unde: Descendit in hortam nucum. Nux enim dulcem fructum habet intus, qui nisi fracta testa non ostenditur loris: sic sanctorum vita suavitatem gratiae in corde conservat. quae non cognoscitur extorius nisi. soluto carnis domicilio animae, in coelesti luce sose conspiciant".

${ }^{6}$ Enarrationes in Cantica Canticorum, 162, 1218C: "(...) in nuce habetur exterius amaritudo, intra testa quaedam fragilis, intus ost nucleus dulcis, eodem modo sancti exterius amaricationes multas sustinent intra corpus, quod est testa fragilis; verum intus quidem est dulcissimum, quod est anima ornata multis virtutibus".

"il Commentaria in Cantica Canticorum, 206, 659C: "I Iujus nucis est hortus Ecclesiae, in praclatis vero cortex est dejectio, testa potantia, corium voluntas, nucleus sciontia. Dejectio, ne sit elatus; potentia, ne in co sit defectus; voluntas, ut in co sit affectus; scientia, ut sit gnarus".

${ }_{62}$ Expositio in Cantica Canticorum, 164, 1275A-13: Descendi in hortum nucum. Quid per hortum nucum, nisi Ecclesiam? Cur autem Leclesia hortus vocetur (...) nucum? Nux enim suavem reddit odorem. Ejus autem fructus duplici tegmine induitur. Quod autem exterius est, amarum est; secundum autem tenax et durum. Quod vero intrinsecus latet, dulco est atque suave. Sic autom Ecclesia exteriorem vestem, carnem vidalicet amaram, miseram, lacrymabilem, horridam et hispidam habet. Sub hac autem illa fortis, candida, et unica tegitur vestis quam ipsa suis manibus texuit: virtutes enim virtutibus conjungens. operibusque bonis bona opera connectens, admirabilem sibi composuit vestem; qua induta, inimicorum spernit sagittas. Sub hac autem tegitur anima, mira dulcedine et suavitate, follis omnisque amaritudinis expers". 
Honorio de Autun, en diversos pasajes $s^{13}$; Gilberto Foliot ${ }^{\text {int }}$; Garnerio Lingone$n e^{65}$; Tomás Cisterciense/Juan Algrino, en diversos pasajes ${ }^{56}$; Alain de Lille ${ }^{67}$.

${ }^{53}$ Expositio in Cantica Canticorum: a) 172, 454A-B: "In nuco tria notantur, cortex exterior, qui est amarus, testa interior, quae est dura, el enuclear quod est dulce. Cortex sunt hi, quibus amara sunt vitia; testa, qui frangunt corpus vigiliis at jejuniis; enuclear vero, spiritales viri"; b) ibid. , 461 B-C: "In nuce tria considerantur, scilicet amurca amara, testa dura, el nucleus dulcis. Per haec notantur tres ordines in Ecclesin; per amurcam conjugati in tribulationlbus mundi amaricati; per testam continentes contra vitia duri; per nucleum virgines in virtutibus dulces"; c) Specalum ecclesiae, 172, 839D: "Iortus est Ecclesia, nuces sunt divorsa sanctorum agmina. In nuce tria considerantur, per quac tres ordines in Ecclesiat designantur. Per exteriorem scilicet corticem tonjugati, per testae fortitudinem praelati, per nuclearem dulcedinem contemplatione indulcorati denotantur. In hunc hortum ad tales nuces Dominus descendit cum de coelis in hunc mundum in humana forma venit".

it Exposilio in Cantica Canticorum, 202, 1287A: "Quid istue nuces significant? Corda utique sanctorum, quae quidem interius, nuclei dulcedinem, id est, spiritus utilitatem praeferunt; exterius vero carnis utilitatem ostendunt, quac adeo dura est, ut de a dictum sit, Labemus huncthesarum absconditum in vasis fictilibus (II Cor. IV,7)",

${ }^{15}$ Sermones. Sermo XXI, "In dic Sancto Pentecostes", 205, 706A-B: "Quia vero scyphi in modum nucis fuisse dicuntur, aliquid ad nucis similitudinem habere tenentur qui verbo ot exemplo aliis praeesse videntur. llabent enim in extoriori testa, quod [rangi debet per abstinentiam; babent extra corticis amaritudinom, quae tolerari debet per patientiam; habent et nuclei dulcedinem. spem salutis, quao dulcorat animum per devotionem. Quod autem tres scyphi, vel tres spherulae, vel tria lilia per singules calamos fuisse perhibentur, hoc figurat, quod praedicatores, qui verbo et exemplo alios inebriant, lıujus debent esse discretionis, ut sciant discernere quan quibus dubuant pradicare".

the Commentaria in Cantica Canlicorum : a) 206, 477C: "Ut primo vescamur, prius ferro desecatur, secundo in olle ponitur, tertio in igne coquitur: secundum, scilicet pomum, decoriatur; tertium, id ast nux a sua testa confracta separatur, quartum, id est aroma mortario contunditur. Primi sunt poenitontes, secundi exteriorem favorem suorum quasi pomi scoriam abjicientes, tertiisunt confractionem aflictionum voluntarie sustinentes, quarti sunt illatas passiones imbuti superno sapore non sentiontes, et inde in aliis bonum odorem largientes"; b) ibid., 608B: "Nuces sunt in horto licclesias, qui militant sub passionum gravitate; poma convallium. qui se cohibent in humilitate; vineae, ferventes in charitate; mala punica, conjuncti unanimitato"; c) ibid., 655C-D: "I [ortus isto est Feclesia ín quo sunt nuces, id est justi. Recte nucibus comparatur vita justorum, quia ita suavitatem fructus spiritalis intimo corde sorvant, ut banc proximis quanta sit for is innotescere nequeant. Item sicut nux amarissimum habet corticem et testa durissima accingitur, et detractis austerioribus et duris, fructus dulcissimus roperitur. sic omnis corroptio ot labor continentiac, qua santa exercotur Ecelesia, amara quidem videtur ad praesens, sed fructum parit in [uturo dul cisstmum"; d) ibid. , 668B: "Siquidom Ecclesia per hortum nucum designatur: propter patientiam sanctorum et fructum pationtiae, quae signatur in nuce, quae sub amaro cortice et testa dura, dulcem continet nucleum, sic patientia per amaritudinem passionum et constantiae robur, pervenit ad dulcedinem praemiorum".

${ }^{67}$ Distinctiones dictionum theologicalium,210, 878B-C: “Nux proprie. Dicitur martyr, unde in Cant. (...). Nux tria habet in so: putamen exterius, testam et nucleum: similitor martyr tria habet: defoctus carnis quasi putamen, carnom quasi testam, animam quasi nucleum. In fractura autem nucis projicitur putamen, testa frangitur. 
2.15. Nuez $=$ los Sacramentos: 5 . Bernardo ${ }^{\text {r.t. }}$.

2.16. Nuez $=$ Cristo. En este campo, las simbolizacioness son numerosas y en ellas podemos distinguir dos tipos: a) las que se refieren a Cristo, nacido de una Virgen simbolizada por la vara de Aarón, y b) las que se refieren a Cristo directamente.

a) "Auctor incertus" (= 5. Agustín? \}", en un texto que es repetido, al pie de la letra por Anselmo Laudunense ${ }^{70}$; Salonio Viennense ${ }^{71}$; Remigio Antisiodorense ${ }^{72}$ Pedro Damián ${ }^{73}$; Wernero "S. Blasii" ${ }^{\text {"74 }}$, en un texto repeti-

nuclei dulcedo gustatur: sic per martyrium defectus desinunt esse in martyre, caro quasi testa quodammodo Irangilur, et tunc in anima sanctus dulcedine aeternae beatitudinis delectatur". (Mlain es el único autor, de ontre aquellos a los que nosotros hemos tenido acceso, que, para reforirse a la cáscara exterior de la nuez, utiliza al término putamen).

${ }^{68}$ In coena Domini. Sermo "De baptismo, sacramento altaris et ablutione pedum" 183, 271 B: "Sic et ego vobis, fratres charissimi, si possom, sacramenta quae clausa sunt, aperire debueram; sed, qui minus possum, rogemus ut vobis pariter tot mihi mater sapientia [rangat nuces jstas, nuces, inquam, quas protulit sacerdotalis virga, virga virtutis, quam emisit Dominus ex Sion".

(4) Sermones. Sermo CCXLV, "De mystorio Trinitatis ot Incarnationjs", 39, 2197-98: "Virga illa (...) Aaron Maria luit (...). Quod ergo baec virga nuces produxit. imago Dominici corporis fuit. Nux enim trinam habet in suo corpore substantiae unionem, corium, tostam et nucleum. In corio caro, in testa ossa, in nucleo interior anima comparatur. In corio nuris carnem significat Salvatoris, quas habuit in se asperitatem vol amaritudinem passionis. In nucleo interiorem declarat dulcedinem deitatis, quas tribuit pastum, et luminis subministrat afficium. In tosta, lignum interserens crucis, quod non discrevit. id quod foris et intus fuit, sed quate terrena ot coelestia luerunt mediatoris ligni interpositione sociavit (...)".

"Epistola ad II. Abbatem S. Laturentii Leodiensis, Pal. Lat. 162, 1592A-B.

"En una simbolizarión de amygdalum y nux amygdali: Expositio mystica in Ecclesiasten : 53, 1009D-1010A: "per umygdalum destgnatur Dominus Jesus Christus: quia sicut nux amygdali constat ex corio, osse et nucloo, ita Christus ex carne, anima et divinatione".

72 También aquí, con una simbolización a travós de amygdalum: J/omiliac. "Homilia IV", 131,888D; "Et bene Dominus noster Jesus Christus similatur amygda]o: nam sicut amygdalum constat ex usse, cortice; et medulla, ita Dominus noster Jusus Christus constat ex divinitate ot humanitate, carne scilicot ot anima".

${ }^{73}$ Èn un texto donde aparece la expresión amygdalinas nuces: Sermones. XLVI. IIomilia "In Nativitate Beatissimae Virginis Mariae”, 144,760B-C: "Et bene virgula fumi hace dicitur quae per virgam quoque Aaron mystice figuratur. Illa enim anygdalinas nuces absque ullo humore terreni cespitis protulit; ista vero absque ullo virili somine Dei Filium generavit. Ipse quoque virgae illius Iructus, si studiose discutitur, aliquam cum Christo babere similitudinem invenitur. Sicut enim amygdalina nux ox nucleo constat, osse et cortice; ita redemptor noster ex divinitate constat, et anima simul et carne".

th Libri deforationum, 157, 793 A-B: "Similiter virga Aaron quae sine semine protulit nuces, significat Mariam, quae sine semine genuit Christum; in nuce, quac significat Dominicum corpus, tria sunt: cortex, testa, nucle us. Cortex amarus significat carnem, quae habuit passionis amaritudinem. Testa significat ossa; nucleus interior, animam candidam virtutibus". 
do, al pie de la letra por Hildeberto de Lavardin ${ }^{75}$; Honorio d"Autun ${ }^{76}$; Amedeo Lausanense ${ }^{77}$; Adam de san Victor ${ }^{74}$; Ruperto Tuitiense ${ }^{77}$; Hono-

${ }^{75}$ Sermones. Sermo "In Nalivitate Domini, Sermo tertius", 171, 3934. Por su parte, Hildeberto, en otro pasaje (Sermones. XLIII, "In die Sancto Paschao. Surmo primus", 171, 559C), olrece una simbolización a traves de amygdalas: "Anygdalus namque tria habot, corticem, tostam, nucleum; et Christus in tribus vere subsistit substantis. In co quippe est cortex carnis, testa mentis, nucleus deitatis". El mismo autor, en una composición en dísticos elegíacos, expone bien a las claras la simbolización: la vara de Aarón dio [rutos ("nuces"); la Virgen dio a luz al HombreCristo, simbolizado en la nuez: Carmina miscellanea, tam sacra quam moralia. Sive Libellus qui dicitur "Floridus aspectus", II: "De partu virgineo" (171, 1383C-D):

"Aaron virga, Dei Virgo peperisse Toruntur;

Arboris illa nuces, netheris ista Deum.

Semine virga caret, profert sine semine fructum;

Nescit Virgo virum, concipit absque viro.

Fert sine radice, genuit sine seminis usu

Arida virga nuces, integra Virgo Deum.

Floruit illa, dedit fructum, nux proditit inde:

Concipit, ista parit, nascitur inde Deus.

De virga nux exoritur, de Virgine Christus;

Virga Maria luit, nux IJomo-Christus erat.

In testa sane species humana notatur.

In nucleo deitas, in nuce Christus-1 lomo.

In testa latens deitas, in carne moratur

Cum nucleo testa, cum dejtate caro.

Dulco sapit rucleus, mulcet iste palatum,

Dulce sapit deitas, cor regit iste sapor".

7"En una simbolización de amygdalum: Quaestiones in Proverbia et Ecclesiaslen, 172, 34613: "nam per amygdalum designatur Dominus noster Jesus Christus".

${ }^{77}$ En una simbolización de amygdalum: Homiliae de Maria Virginea Matre, 188, 1308B-C: "Illa (= virga Aaron] fructum protulit amygdalinum, ista [= Virgo Marial et optimum protulit amygdalum, habentem nucleum et testam. Nucleum ut reficiat; testam, ut contegat. Nucleum in Divinitate; estam in humanitate. (...) Et quoniam testa etiam corticem habet, corticem intellige in carnis amaritudine, tostam in resurrectione, nucleum in deitate".

${ }^{7 s}$ Sequentiae, III: "Dominica intra octavas Nativitatis Domini", 196, 1432B ss.: vers. 25 ss.: "Frondem, florom, nucem, sicca/Virga profert; et pudica/Virgo Deí Øilium"; vers. 35 ss. : "Flos dulcore, nux pascendo (...)/Sicca sic amygdalum"; vers. 47 ss.: "Nux est Christus, cortex nucis / Circn carnem poena crucis. / Testa, corpus ossium. / Carne tecta deitas, / Et Christi suavitas, / Signatur per nucleum".

${ }^{70}$ De Trinilate el ejus operibus. In $\alpha$ Numeros, Jib. IJ, cap.4, (167,882D-883A): "In illa virga speciosae novitatis Evae genus reviruit, in illo nore quem peperit, carnis nostrao silva refloruit, in illa nuce quassa vel fracta per lignum crucis anima nostra nucleum vitae invenit".

${ }^{56}$ Speculum ecclesiae, 172, 850B: "Virga $\Lambda$ aron arida, sed fructu horida, est sancta Maria, virginitate quidem arida et Spiritu sancto fecundata et partu gravida. Haec virge dulcem nucem adidit, dum Virgo Deum et hominem genuit. In cortice quippe nucis caro Christi. in testa ejus ossa, in nucleari ipsius anima notatur; vel exteriorem corticem humanitatis ipsius. Per nuclearis dulcedinem ejus divinitas 
rio d'Autun"; "Adamus Perseniae"s!.

b) Paulino de Nola ${ }^{H 2}$; Salonio Viennense ${ }^{83}$; este texto lo repite, al pie de la letra, Honorio de Autun ${ }^{84}$; este último autor, en un texto propio ${ }^{85}$; san Bernardo ${ }^{\mathrm{A5}}$; Guerrico Igniacense ${ }^{87}$; Pedro Celense ${ }^{\mathrm{k8}}$; Thomas Cistercien-

declaratur. Nux quodam interliminio in modum crucis funditur lleg. finditurl, et anima Christi a corpore ejus cruco dividitur".

"1 Mariale. Sermo primus: "In annuntiatione B. Virginis», 211, 707A-B: Fronduit, quando obumbrans el Altissimi virtus, et angelis, et hominibus mysterium occultavit. Fructificavit, quando quem sine contagione conceperat. sine dolore partu edidit. Conceptum itaque Virginis virgae florem dicimus, velamenta mysterii frondes, partum nucem de virga prolatam. Nux optime convenit sacramento. Denique nascentis unam in tribus substantiis personam figurat. Corium nucis refer ad carnem, testam ad animam nucleum, ad interiorem internae divinitatis dulcedinem. Corium habet amaritudinem, caro Salvatoris sustinuit passionem. Testa fortior animae mundum salvantis designat virtutem". 654C-D:

${ }^{82}$ Poemata. Poema XXVII: "De S. Felice Nalal", carmen IX. Pat. Lat., 61.

"Spiritus in platano est, Virgo in storace, in nuce Christus.

(...)

Virga nucis Christus, quoniam in nucibus cibus intus

Testa Toris, et amara super viridi cute cortex.

Corne Deum nostro velatum corpore Christum,

Qui fragilis carne est verbo cibus, el cruce amarus.

Dura superficies, verbum crucis; el crucis esca est.

Coelestem Clsristi claudens in carne medullam".

${ }^{*}$ En un texto donde encontramos amygdalum, por un lado, y nux amygdali por otro: Expositio mystica in Ecclesiasten, 53.1009D-1010A: "Haec sententia ]= Et forebit amygdalus... I spiritualiter est intelligenda, et magnum in se continet mysterium. Nam per amygdalum designatur Dominus Jesus Christus: quia sicut nux amygdali constat ex corio, osse et nucleo, ita Christus ex carne, anima ot divinitate. Quoniam per os amygdali divinitas designatur".

Ht Quaestiones in Ecclesiasten, cap. XII, $(172,3468)$.

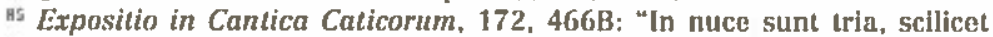
amurca, testa el nueleus; ita in Christo sunt tria, videlicet caro, ossa ot anima. Interstitium quod nucem dividit in modum crucis, est sancta crux, quae animam Christi a corpore divisit".

${ }^{86}$ Sermones. Sermo "In Dominica I postoctavam Epiphaniae", sermo secundus, 183, 158A-B: "Totus $1=$ Christus 1 suavis est (...). Nam et superfictes ipsa, tanquam a foris considerata, decora est valde, et si quis fregerit nucem, intus inveniot quod jucundius sit, et multo amplius delectabile".

${ }^{87}$ Sermones per annum. "De resurtectione Domini", Sermo I, 2, (185, 142A): "Ovum sive nucem apposui vobis, Iratres; frangite testam, et invenielis escam. Joseph discutiatur, et Jesus invenietur agnus paschalis; qui tanto dulcius comeditur, quanto latens abstrusius, et studiosius quaeritur, et diflicilius invenitur".

${ }^{88}$ Liber de panibus, cap. VI $(202,955 \mathrm{D})$ : "Qui orgo non tanquam asinus in stercore suo piger, fenum luxuriac comedit, non velut jumentum luxurians, herba vanitatis relicitur, sed cum pontifice Aaron, panem ex adipe frumenti, sed cum Jesu in cruce passo corio detracto el testa confracta, nucleum nucis, medullam olei, vini et frumenti, in sortem is accepit". 
se/Juan Algrino ${ }^{89}$; Alain de Lille, en una doble interpretación de la nuez como símbolo de Cristo"e.

2.17. Nuez = la Cruz del Señor: sam Ambrosio" .

2.18. En algún caso, los autores dudan a la hora de aplicar un tipo de simbolización: así, Rabano Mauro, a continuación de un pasaje ya citado, ve en las nueces el símbolo de la Encarnación del Salvador o, como quieren algunos, el misterio de la santa Trinidad ${ }^{92}$.

Como vemos, un intrincado laberinto de simbolizaciones de todo tipo, fiel reflejo de un mundo que, en manos de sermoneadores y predicadores, intenta descubrir la transcendencia en las más variadas circunstancias de la vida. Y es que, a lin de cuentas, de lo que se trata es de descubrir el misterio y el auténtico sentido de las cosas, saber leer en ese gran libro que es el mundo sensible, como hermosamente lo dejó dicho Hugo de san Víctor ${ }^{33}$ : "el mundo es una especie de libro escrito por el dedo de Dios".

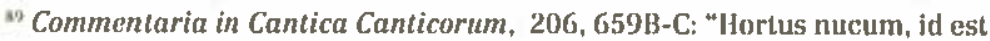
praclatorum, dicitur Excclesia, qui nuces habere dicuntur hac ratione. Nux labet quatuor quac ad praelatos pertinent: exterits vilem et amarum corticem, secundo testam, tertio corium. In Christo cortex fuit humjlitas et amaritudo conversationis, testa caro ejus fortis contra incentiva libidinis, in corio anima, in nucleo divinitas".

${ }^{90}$ Distinctiones dictionum theologicalium, 210, 878C: a] "Nux otiam [unas" líneas más arriba ha hablado de la nuez como simbolo del martir, como ya lo hemos hecho notar] solet dici Christus, quia in Christo fuit corpus quasi putamen exterius, anima quasi testa, divinitas quasi nucleus". b) "Quandju Christus mansit in mundo, divinitas non apparuit in co, sed in corpore separato ab anima post mortom quasi [acta ["sic" por "fracta"] testa, Jomo sensit dulcedinem nurlei, quia liberatus per divinitatis potestatom".

"De Joseph Patriarcha, 14, 661в: "Venit enim Paulus crucem Domini praedicare, ilicem semper virentem, et nuces quarum testa durior, fructus tenerior: meritoque virga sacerdotalis Aaron nucina, et Hieremitse baculus hujusmodi".

${ }^{\prime 2}$ De universo, lib. XIX, cap. 6, (111, 514B): "Item nuces incarnationem Domini Salvatoris, sive mysterium sanctue Trinitatis (ut quidam putant) significant". ${ }^{3}$ Erruditio didascalica, VII 4 (176, 814B): "Universus enim mundus jsta sensibilis quasi quidum libar est scriptus digito Dei". 